\title{
Design of Morphing System for Anthropomorphic Animal Characters
}

\author{
Young-Suk Lee ${ }^{1}$, Sang-Nam Kim ${ }^{2}$, and Sanghun Park ${ }^{3}$ \\ ${ }^{1}$ Institute of Image and Cultural Contents, Dongguk University, Seoul Korea \\ ${ }^{2}$ Graduate School of Digital and Image, Dongguk University, Seoul Korea \\ 3* Department of Multimedia, Dongguk University, Seoul Korea \\ tonacoco@dongguk.edu,naminamiya@naver.com, mshpark@dongguk.edu*
}

\begin{abstract}
In this paper, the classification of animal facial characteristics is examined for the purpose of creating anthropomorphic animal characters, followed by a description of the design process involved in creating a morphing program that can be used to generate various anthropomorphic animal characters. In order to establish an animal archetype database $(D B)$ and an anthropomorphic animal character $D B$, we systematically analyze the facial characteristics of mammal characters. Subsequently, a technique is proposed with which to express component-based animal faces using a B-Spline curve in the morphing system.

This program offers an intuitive user interface with a prototype generated upon the definition of necessary menus that allow system users to select optimally anthropomorphized images from an input source-target image morphing process.
\end{abstract}

Keywords: Classification of animal characteristics, Design of morphing system, Anthropomorphic animal characters

\section{Introduction}

New coding education, which started with elementary schools in the U.K. [20], is spreading worldwide. In particular, the "Scratch" program developed by researchers at the MIT Media Lab is helping elementary school children easily learn programming principles (htpp://scratch.mit.edu). Using this program, children can, for example, simply select and drag code blocks to cause a feline character named Felix to move across the screen. Here, an animal character is used to enhance programming intuitiveness. More specifically, young children's interest and intuitive sense for programming is enhanced with the use of an animal character. The rising demand for characters is no longer limited to the fields of animation or gaming. In addition, the role of characters is only expected to grow, as is the demand for characters, through the expansion of the digital content industry. It is also anticipated that the character industry, which was previously limited to the production of character products and animation, will expand into various other industries. Furthermore, the growing supply of smart media is setting a new trend where local government entities, venture companies, and even individuals can participate in content production, in addition to the regular professionals in the industry. Development of such ultra-niche markets predicts a continuing expansion of hyper specialized content that is produced by the community and geared to different consumer ages and tastes [16]. The production of digital content geared toward specific markets, (e.g., content appropriate for infants) is gaining momentum. In particular, prolific consumption of launcher applications, known as the user interface/user experience (UI/UX) of smartphones is highlighting the growing role of the character industry in the mobile domain. In the marketing domain, businesses rely increasingly on the benefits that characters provide for expanding brand value [1]. This is because it is particularly more 
advantageous to adopt characters to market products with a high rate of consumption [18]. According to Bettany et al. [2], animal characters are often favored among various character options because animals that behave similarly to humans can facilitate a creative escape from reality more effectively than their human counterparts. Because of the recent surge of content supply and demand caused by the expanding smart media market, authoring tools are being actively developed in various domains. In the scenario domain, the development and utilization of authoring tools are already well established. Game and animation industries are demonstrating an increased interest in authoring tools, which is expected to make the design process more effective. As such, research is in progress to develop new motion by analyzing the existing motion of animated characters through video clip sampling [3]. Authoring tools for creating anthropomorphic animal characters have not yet received much interest. However, such tools should be developed in order to increase the efficiency of cost and labor required for character development. The study of face types is being standardized in the field of facial recognition technology. Criminal investigation and applications related to image surveillance use this technology, where captured images analyzed in a database (DB) to find a match and to identify people. Face type research in the field of content is centered on animation characters, most of which explore the human expression of emotions. On the other hand, studies on the face types of anthropomorphic animal characters are few. Furthermore, the objectives of existing research are limited to the analysis of face types or emotions of existing animal characters without concern for the development of new anthropomorphic animal characters, in general. As such, a detailed analysis of human and animal face types is required in order to develop various anthropomorphic animal characters. Our study aims to develop a morphing program based on the analysis of facial characteristics of people and animals, which will help create a pool of diverse anthropomorphic animal characters.

This part of our study consists of preliminary research required for developing a visualized automated morphing program with which to create anthropomorphic animal characters. Study objectives are as follows.

- Facial features and characteristics of animals are analyzed and compared for the purpose of establishing a set of standard facial characteristics for anthropomorphic animal characters. Components of facial characteristics are also determined to allow smooth and natural morphing of human and animal faces.

- Technology for implementing optimal curved surface forms is investigated for effective morphing. In order to achieve natural morphing of anthropomorphic animal characters and humans, the curves and straight lines between feature points are addressed. The B-spline curve technique is used to obtain an effective curve at each critical point.

- An intuitive manual is created for system users. UI is designed in ways that appeal to the users' intuitive sense for the purpose of establishing an anthropomorphic animal character morphing system - the fundamental goal of our study. A prototype is designed upon extraction of the minimum number of required functions, the purpose of which is for future research to complement the findings obtained in this study.

First, we analyze the facial features and characteristics of various animals to establish a set of components. Thereafter, the critical facial feature points of humans and animals are matched carefully to obtain natural morphing results that are used in the subsequent design process of a morphing program prototype of anthropomorphic animal characters.

\section{Study Trends in Facial Characteristics of Characters}

In this study, we aim to design a program prototype that can create automatically a character face type desired by a user through the symmetrical analysis of animal and human facial characteristics. First, we examine animal facial features and characteristics to compare with those of a human. Literature analysis of existing research is also 
conducted for the purpose of creating a prototype for an automated anthropomorphic animal character morphing system.

\subsection{Study of Anthropomorphized Animal Characters}

Anthropomorphism refers to the tendency for imbuing real or imagined behavior of nonhuman agents with humanlike characteristics, motivations, intentions, and emotions [4]. In the field of digital content, the demand for anthropomorphic animal characters is increasing because of their capacity to offer an easier escape from reality compared to their human counterparts, as mentioned previously [2]. Research on anthropomorphic animal characters is at a relatively nascent stage. In Korea, animal character studies only began in the 2000s. Previously, studies on anthropomorphic animal characters were limited to those appearing in literature and paintings for symbolism analyses. Only in recent years has the appearance and other outward characteristics of animal characters start to garner the attention of researchers. According to a 2003 study by S.-W. Park on the motives behind anthropomorphism in character design, empathy and isomorphism were found to be major motives for anthropomorphism [5]. In 2009, Y-S, Lee engineered the steps for animal anthropomorphism using the external appearance and characteristics of animal characters [6]. Lee classified criteria for such characteristics based on animal taxonomy, and organized the characteristic feature points of each animal. In 2009, S.-H. Song studied the visual language expressed in anthropomorphized animation characters. Upon formative characteristics analysis paired with psychoanalysis, a character's personified psychology expressed through colors was found to be identical to the general psychology of colors. In addition, it was found that individuality was assigned through added chromatic sense on a small surface. Anthropomorphic characters are accorded conflicts and other human conditions of our society while simultaneously expressing emotions and imagination [7]. In 2013, S.-Y. Sohn studied characters used in advertisement, through which she discussed the effectiveness and characteristics of TV commercial characters through analyses of character types and case studies. [8]. In other countries, the correlation between anthropomorphic characters in children's literature and the social learning of children, as well as anthropomorphic components, have been studied mostly in the field of psychology [9-10]. Research on the visual representation of anthropomorphic characters is being conducted. However, such research lacks variety and its scope is limited to classification. In Korea, further research is also required for various potential industry applications of anthropomorphic animal characters.

\subsection{Facial Definition Parameters}

Facial recognition technology is used to recognize and identify human faces using only the images entered into a computer. First, the technology extracts the facial parameters and analyzes them in a DB to match and identify a person. Application of facial recognition technology can be found in biometrics, information security, smart cards, etc. In particular, the international standard Facial Animation Parameter (FAP) is a collection of feature points used to synthesize animated characters' facial expressions. The points are marked in MPEG4, which is expressed through a Facial Definition Parameter (FDP) and FAP. We believe that the adoption of feature point use is also required to create anthropomorphic animal characters.

Studies on embodiment and synthesis using facial characteristics have traditionally concerned the human face. While using images obtained with a single camera, V. Blanz, and T. Vetter (1999) established a 3D face model that could be manipulated. However, this model required a significant amount of time to reconstruct a face because of numerous calculation processes [11]. S. Wang et al. (2006) and C. Wang et al. (2003) conducted research on a 3D face reconstruction model that can be manipulated with face form data. However, result accuracy depended greatly on the accuracy of the extracted 
facial feature points. Other limitations include the fact that facial characteristic extraction using an extraction tool does not properly accommodate changes in lighting conditions and other factors [12-13]. The facial feature points of anthropomorphic animal characters used in this study are based on the animal taxonomy and characteristics described in Y.-S. Lee's study $[6,19]$. Common animal facial features are classified and defined to facilitate effective comparison and matching with a human face. The study by Lee et al. [6] is used as the basis for our research to design our proposed program in practical terms. Image morphing refers to special visual effects that sequentially change one image to another through a frame level warping process. This technology is most commonly used to morph two separate facial images into one. Such morphing can be categorized into two criteria: feature point-based morphing, and mesh morphing.

Feature point-based morphing is relatively time consuming because it is performed in pixel units on control parameters composed of facial feature points. However, various manipulations are possible and users can complete their job according to their requirements. Mesh morphing, which facilitates simple and easy processes and saves time, requires mesh control points to be manually adjusted. Our objective is to extract various resulting values through specified facial feature points of people and animals. For this reason, feature point-based morphing is used in this study. N. Kwak [14] established a field based semi-automated morphing technique where users set control parameters and define the feature points with outside input for both the source and original image. V. Zanella et al. [15] employed the Active Shape model, which uses predetermined face models in order to extract facial characteristics and automatize image morphing. To ensure a smooth connection between feature points, B-spline is used in this study. Bspline curve fitting refers to the production of a smooth curve that passes through different sets of coordinates $\mathrm{Pi}(\mathrm{i}=0, . ., \mathrm{m})$ [17]. The B-spline curve consists of polynomial control points that include the desired curve, and a blending function that combines the effects of the control points.

\section{Classification of Anthropomorphic Animal Character Feature Points and System Configuration}

\subsection{Classification of Anthropomorphic Animal Character Feature Points and Suggestions}

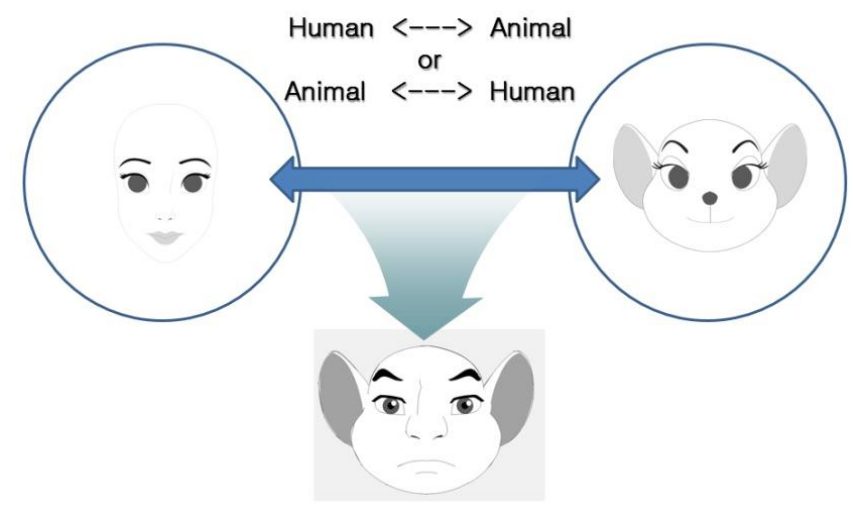

\section{Figure 1. Authoring Tool that Allows Creation of Diverse Character Forms}

Our study aims to plan and design a character morphing program that allows character designers and general users to easily create animal characters. In this chapter, we describe the animal DB configuration, a critical component of the anthropomorphic animal 
character morphing system. In order to produce a variety of animal characters, a DB was configured based on 60 types of mammal archetypes referenced in animal taxonomy and on anthropomorphic animal characters that appear in animated feature films made in the U.S. The DB created for this study is capable of suggesting transitional characters that possess average morphed characteristics of the select animals and humans [Figure 1] Such feature allows character diversification with data input from a single character. To allow this, our study provides a DB based on 60 anthropomorphic animal character types. For an effective DB, we suggest a set of common denominators between human and animal characters, and define it as anthropomorphic animal character facial feature components.

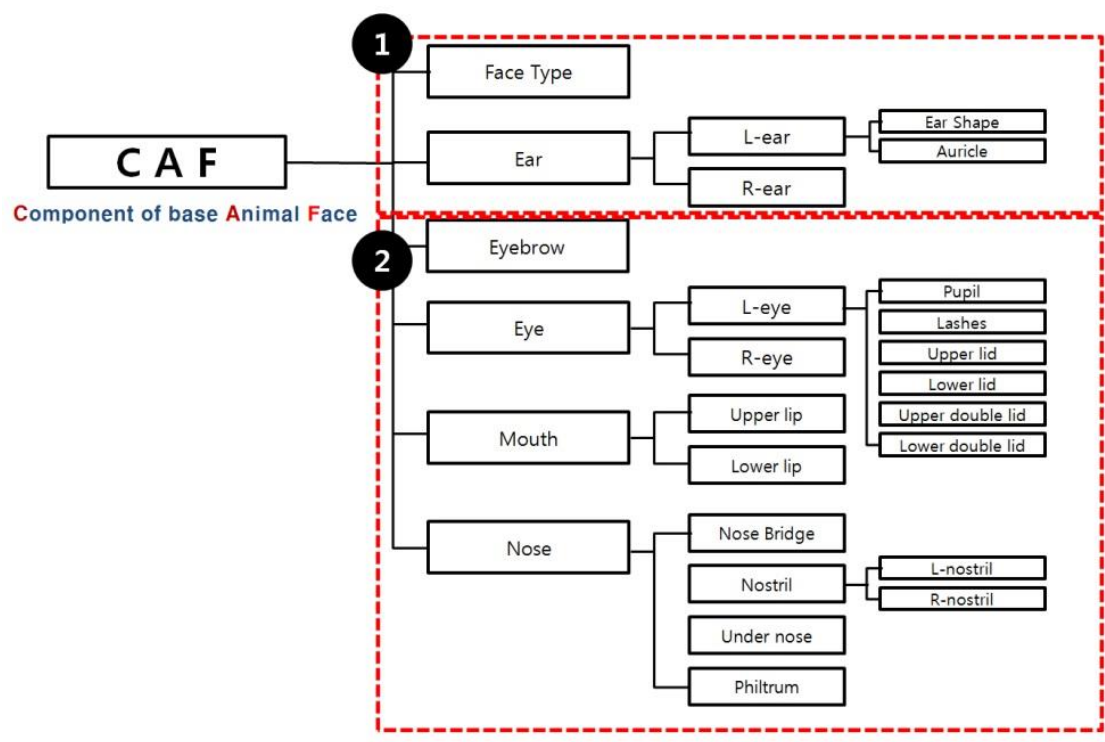

Figure 2. System Summary

The overall system summary is as follows.

First, a user enters an image or selects input data from the anthropomorphic animal character prototype DB, as well as one of the characters stored in the system. Subsequently, the system analyzes the data and finds matching components for linking [FIG 2]. To implement the system, we first analyzed previously defined animal characteristic types based on the B-spline curve.

In this section, we examine animal types in detail according to taxonomy through literature analysis of prior studies. In order to design characteristic components of animal characters, we must classify such characteristics according to animal taxonomy, and subsequently extract the frontal facial features and characteristics of animals. However, for this study, frontal facial feature characteristics are obtained from prior studies. Although the mammal types suggested in animal taxonomy are incredibly diverse in terms of appearance, common characteristic components are present.

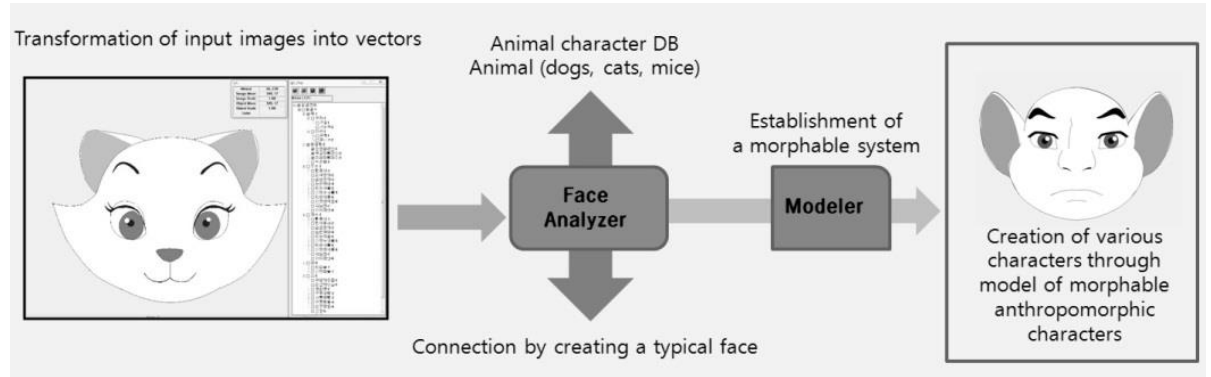

Figure 3. Composition of Anthropomorphic Animal Characters 
Basic animal characteristics based on taxonomy can be classified as external, ecological, and behavioral. Our study limits the scope to external characteristics, namely, the animal head. Each animal head shares common features with a human head in the form of eyes, nose, and mouth. Additional features unique to animal heads include ivory, tusks, protruding front teeth, ears, and horns. As such, we classified and defined two major components for creating anthropomorphic animal characters: external components unique to animal characteristics, and internal components that consist of characteristics shared with humans. The external components are defined as that which lies outside the contour of the face and includes various face shapes and ears. Internal components include eyebrows, eyes, a mouth, and a nose. When compared with human characters, the basic skeletal features are similar. Common facial characteristics of animals include a prominent forehead bone, the width of the nasal bone under the eyes, protective eyebrows over eyes, and lower eyelids. Components include eyes, nose, and ears. With regard to the eyes, despite the similarity with human eyes, animal eyeballs are rounder and the inner corners are located lower than the outer corners. In terms of the nose, the animal nose is more upturned. The nostrils and the area surrounding the nostrils form a curve (wing) similar to a comma that is greater in length than a human's and more open in shape. This wing-like curve expands in the shape of a flower called morning glory when angered, or when breathing heavily. Pigs have the shortest comma, whereas deer have the longest comma. The most prominent animal nasal characteristic is the septum between the nostrils. It has a groove or is otherwise indented, and it extends to the upper lip. This nasal septum is one of the critical points to consider when selecting characteristic features for creating human and animal characters.

Lastly, ears are featured in a cylindrical shape as shown in Figure 4. Most animal ears are made of cartilage. The cylinder is located below the ears, and in some animals, it is only partially featured. Figure 4 shows a 360-degree rotation view of the ear's frontal image. In camels, cats, dogs, and bears, in particular, the cylindrical shape is hidden.

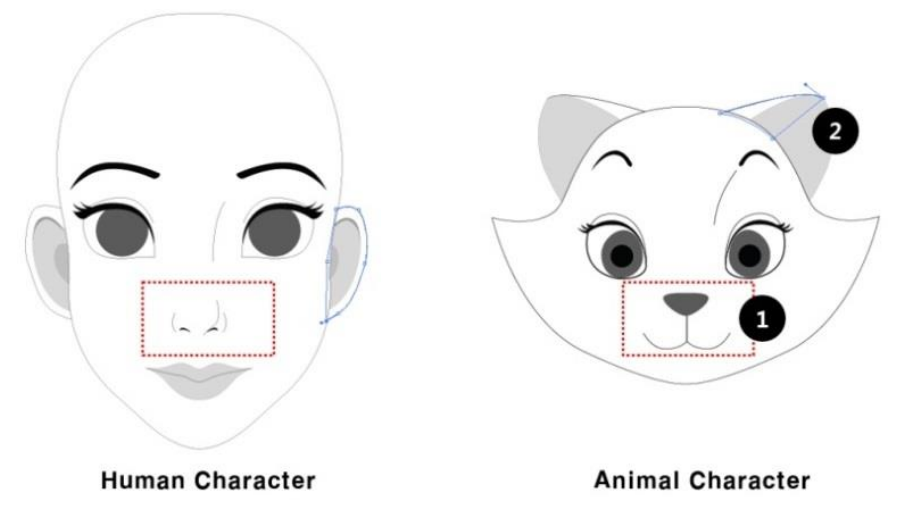

Figure 4. Comparison between Human Character and Anthropomorphic Animal Character

Next, we define the B-spline curve based on the previously classified components. Each B-spline curve has a name and a set of precise coordinates. Each defined curve rotates clockwise, in principle. Subsequently, we consider various shapes. First, the face shape is composed of 14 points to allow various manipulation possibilities. The face shape is called FT(Face Type)_01-14 [Figure 5]. 


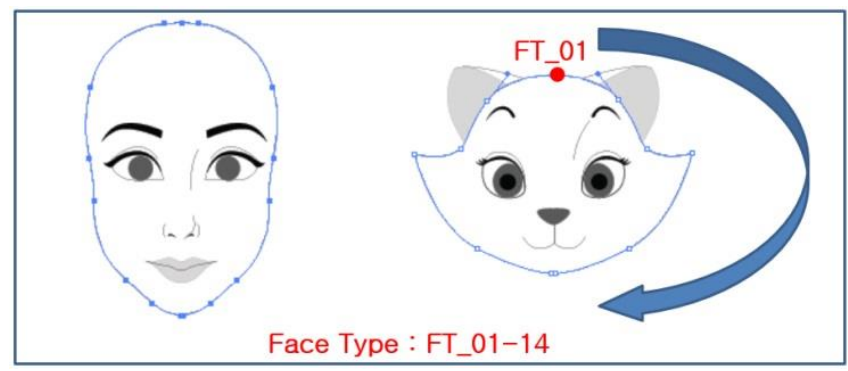

\section{Figure 5. Process of Defining Anthropomorphic Animal Character Feature} Points

Ears are composed of the external and internal ears and are simple in shape. Both external and internal ears consist of five sets of points. The left external ear is defined as EASL_01-05, and the left internal ear as EAAL_01-05. The right external ear is defined as EASR_01-05, and the right internal ear as EAAR_01-05. The eyebrows are composed in pairs, and are divided into left eyebrows and right eyebrows. Because various types of eyebrows need to be considered, 14 sets of points are defined. The left eyebrow is defined as EBL_01-14, and the right eyebrow as EBR_01-14. The eyes consist of the pupils, eyelashes, double eyelids, eyelines, upper eyelids, and lower eyelids. Each consists of eight sets of points. Using the left eye as an example, the eye components listed previously are called LEY_PUP, LEY_LAS, LEY_UPL, LEY_LOL, LEY_UDL, LEY_UDL, respectively. The points for the right eye are defined as REY_PUP, REY_LAS, REY_UPL, REY_LOL, REY_UDL, and REY_UDL.

Considering the movement involved, the mouth consists of 12 sets of points. The upper lip and lower lip are defined as MOU and MOL, respectively. The nose is the most visually distinguishing feature when comparing animal and human characters. The nose is composed of the bridge, nostrils, under nose, and philtrum. These are configured in their entirety, and the nostrils are composed as a pair. Because there is very little movement involved, the nose consists of only eight sets of points. The names assigned to each part are NOBN for the bridge, NO_LNO for the right nostril, NO_RNO for the left nostril, NOUN for under nose, and NO_PH for the philtrum [Figure 3, Figure 6]. We suggested Composition of anthropomorphic animal characters (CAF).

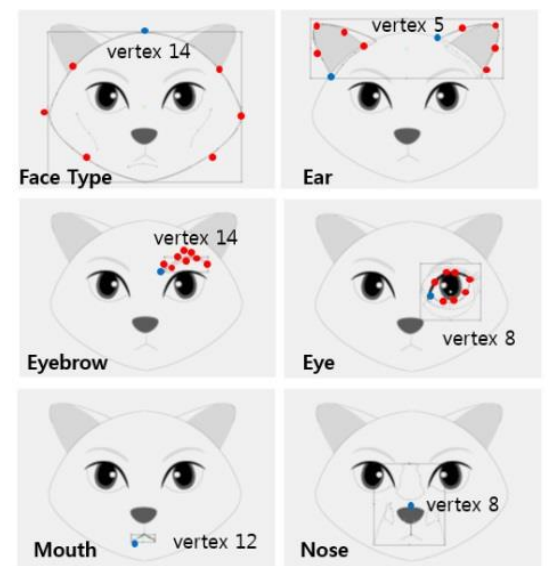

Figure 6. Defining Anthropomorphic Animal Character Feature Point

\subsection{Design Structure of Anthropomorphic Animal Character Morphing System}

Figure 7 shows the practical data processing procedure based on our current design. This system is designed considering user perspective. The system is composed of a user 
input DB and an integrated DB. The integrated DB contains the character data established with the B-spline curve components described in the previous section. Users can execute a variety of animal morphing through the provided DB. The system aims to provide a user-oriented, intuitive UI.

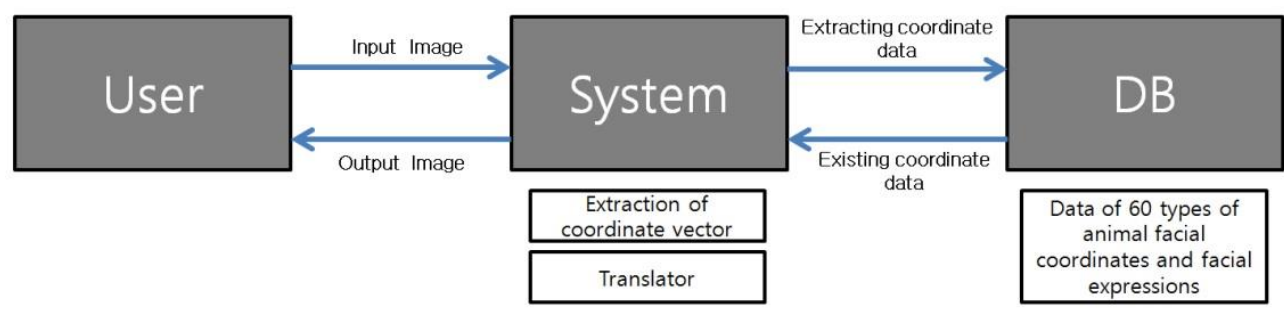

Figure 7. System Data Processing Procedure

A system scenario configuration that considers user perspective is as follows:

1. Import image file

2. Extract face feature points from file

3. Select "cats" type in selection window

4. Click import button

5. Select image from "cats" images imported from DB

6. Verify whether vector coordinates of DB image are stored. If not, select feature points and store vector coordinates in DB

7. For images with existing feature points data, these appear automatically

8. Define distance value between feature points of imported image and DB image

9. Show morphing values in new window

10. Store resulting values or morph again using different distance values

A characteristic feature of this morphing system is that a significant variety of anthropomorphic animal characters can be generated using the internal DB paired with images imported by users.

\subsection{System Configuration and User Interface}

In this section, we suggest a character morphing tool based on the findings obtained from the design process. The UI for data design and processing is as shown in Figure 8.

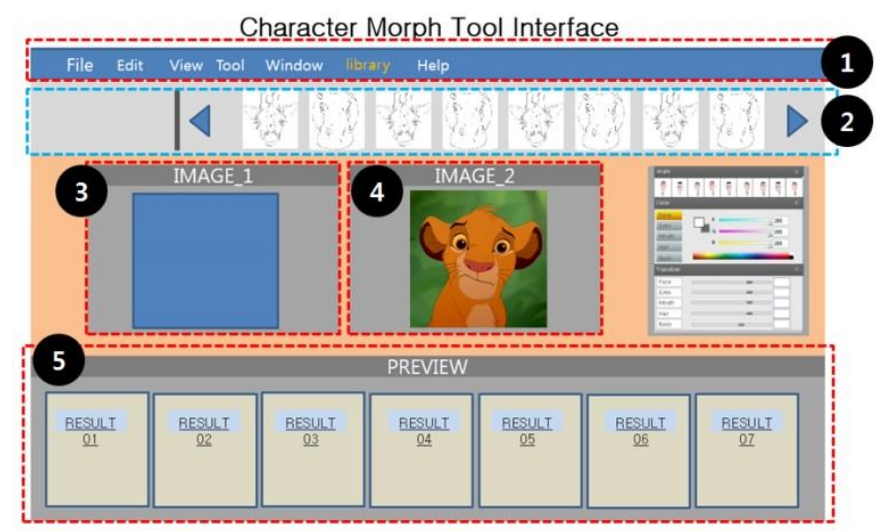

Figure 8. User Interface for Anthropomorphic Animal Character Morphing Tool

The suggested tool allows an effective search for character input data as well as internally stored data. The tool is designed in ways that appeal to the user's intuitive sense 
and allows the user to create a variety of characters. The tool's menu consists of five domains. Domain 1 is the menu bar. Domain 2 is the animal archetype DB provided in the system and the anthropomorphic animal character DB. Domain 3 is the input window where users can import data. Domain 4 imports the DB provided in the system. And lastly, Domain 5 displays the resulting values. The system UI offers two search types. The first type searches the 60-animal archetype DB. The second type searches the anthropomorphic animal character DB. Searching both DBs allow users to select intuitively the morphing features they want.

\section{Conclusion}

This study is a preliminary research for developing an automated morphing program for creating anthropomorphic animal characters. First, we analyzed the facial characteristics of mammal characters in order to establish an anthropomorphic animal character DB and an animal archetype DB. For this, 60 animal characters that corresponded to animal taxonomy were selected. These characters were analyzed for common characteristics, which were subsequently used to propose and define the Component of base Animal Face using the B-spline curve. We compared and analyzed the animal facial feature characteristics in an effort to prepare a set of standards for anthropomorphic animal character faces. In addition, facial feature point components required for smooth and natural morphing between human and animal faces were established. Moreover, the curves and straight lines between feature points were addressed with the B-spline curve technique for effective morphing of human and anthropomorphic animal characters. Next, a minimum number of required functions were extracted and incorporated into a prototype to design a user-oriented and intuitive UI for the purpose of establishing an anthropomorphic animal character morphing system, which is the ultimate objective of our study. This research aims to lay the groundwork for the effective production of anthropomorphic animal characters. As such, our study objective was to analyze the facial characteristics of each animal and firmly establish the key components.

Thereafter, we aim to design an anthropomorphic animal character morphing program prototype that facilitates smooth and natural morphing of human and animal faces through feature point matching. In addition, further studies on anthropomorphic animal character specification standards, including monsters and other species besides mammals, such as birds, should be conducted.

\section{Acknowledgements}

This research was supported by Basic Science Research Program through the National Research Foundation of Korea (NRF) funded by the Ministry of Education, Science and Technology (2014R1A1A1005863)

\section{References}

[1] K. L. Keller, "Strategic brand management: Building, measuring, and managing brand equity", Upper Saddle River, NJ: Prentice-Hall, (2003).

[2] S. Bettany and R. W. Belk, "Disney discourses of self and other: Animality, primitivity, modernity, and postmodernity", Consumption, Markets and Culture, nos. 14-2, (2011).

[3] M. V. de Panne, "Technical Perspective Motion Fields for Interactive Character Animation", Communications of the ACM, vol. 57, no. 6, (2014).

[4] N. Epley, A. Waytz and J. T. Cacioppo, "On seeing human: A three-factor theory of anthropomorphism", Psychological Review, vol. 114, no. 4, (2007), pp. 864-886, doi: 10.1037/0033-295X.114.4.864.

[5] Park, "A Study on the Motive of Anthropomorphic Expression in Character Design", Bulletin of KSBDA, vol. 4 no. 2, (2003), pp. 13-22.

[6] Y.-s. Lee, "Classification and Searching for Anthropomorphic Animal Characters in Animation", Department of Image Information Engineering Graduate School, Pusan National University, S. Korea (2010). 
[7] S.-H. Song, "Study on the personification-oriented visual language of character", Graduate school of Art, Chung-Ang University, S. korea, (2009).

[8] S.-Y. Sohn, "Study on Character Model Used in TV Commercial Film as a CF Model", Korea Society of Design Trend, (2013), pp. 133-134.

[9] S. J. Williams, "Fireflies, Frogs, and Geckoes: Animal Characters and Cultural Identity in Emergent Children's Literature”, New Review of Children's Literature \& Librarianship, vol. 20, no. 2, (2014), pp. 100-111.

[10] Y.-S. Liu, "Inheritance of acquired characters in animals: A historical overview, further evidence and mechanistic explanations", Italian Journal of Zoology, vol. 78, no. 4, (2011), pp. 410-417.

[11] V. Blanz and T. Vetter, "A morphable model for the synthesis of 3D faces", Proceedings of Computer Graphics Proc. SIGGRAPH, (1999), pp. 187-194.

[12] S. Wang, and S. Lai, "Efficient 3D face reconstruction from a single 2D image by combining statistical and geometrical information," LNCS on Asian Conference on Computer Vision, 3852, (2006), pp. 427436.

[13] C. Wang, S. Yan, H. Li, H. Zhang, M. Li, "Automatic, effective, and efficient 3D face reconstruction from arbitrary view image," LNCS on Advances in Multimedia Information Processing, 3332, (2004), pp. 553-560.

[14] N. Kwak, "Semi-automatic field morphing: polygon-based vertex selection and adaptive control line mapping", International JOURNAL OF CONTENTS, vol. 3 no. 4, (2007), pp. 15-21.

[15] V. Zanella, G. Ramirez, H. Vargas, and L. V. Rosas, "Automatic morphing of Face Images., Lecture Notes in Computer Science, Adaptive and Natural Computing Algorithms", vol. 5495, (2009), pp. 600608.

[16] 2014 Contents Industry Prospect, KCCA, S. Korea (2014).

[17] G. Farin, “Curves and Surfaces for CAGD”, Morgan Kaufmann, New York (2002).

[18] M. Costa, "Brand characters: Pulling customers' heart strings. Marketing Week, (2010), p. 16.

[19] Y. S. Lee, S. N. Kim and S. Park, "A Study on Anthropomorphic Animal Characters' Face Shape Features", Proceedings of the 10th International Congress of MITA(2014) July 8-11 ; HKUST, Hong Kong

[20] J. Burns, "School ICT to be replaced by computer science programme", Education \& Family press, BBC, United Kingdom, 2012 (http://www.bbc.co.uk/news/education-16493929)

\section{Authors}

Youngsuk LEE, she is an Assistant Professor at Research Institute for Image \& Cultural Content, Dongguk University. She received B.Des. degree from Tongmyong University in 2002 and Her M.E. \& Ph.D. degrees from Busan National University in 2004 and 2010. Her research interests include the game of graphic design, animation and character.

Sangnam Kim, he is a doctoral student in Culture Contents from Dongguk University. Her research interests include digital storytelling and culture prototype. She received B.A. degree in Literary Creation and B.E. degree in Computer Science from Dongguk University in 2010, and her M.A degree in Culture Contents from Dongguk University in 2014.

Sanghun Park, he received a B.S. in Mathematics from Sogang University, Seoul, Korea in 1993. He also earned his M.E. and Ph.D. in Computer Science from Sogang University in 1995 and 2000, respectively. He is currently a professor at Department of Multimedia, Graduate School of Digital Image and Contents, Dongguk University, Seoul, Korea. His research interests are computer graphics, scientific visualization, and high performance computing. Before joining Dongguk University, he was a research staff member at Computational Visualization Center, Institute for Computational Engineering and Sciences, University of Texas at Austin 